RICYDE. Revista Internacional de Ciencias del Deporte doi: $10.5232 /$ ricyde

Rev. int. cienc. deporte

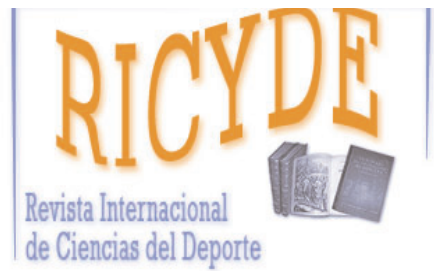

RICYDE. Revista Internacional de Ciencias del Deporte VOLUMEN XI - AÑO XI

paginas:281-296 ISSN : $1885-3137$

Issue 41 - Julio - 2015

\title{
Género y motivación situacional en Educación Física; claves para el desarrollo de estrategias de intervención \\ Gender and situational motivation in physical education: the key to the development of intervention strategies
}

\author{
Javier Sevil ${ }^{1}$, Ángel Abós ${ }^{1}$, Jose A. Julián², Berta Murillo ${ }^{3}$, Luis García-González ${ }^{4}$
}

1. Facultad de Ciencias Humanas y de la Educación. Universidad de Zaragoza.

2. Facultad de Ciencias Sociales y Humanas. Universidad de Zaragoza.

3. Facultad de Ciencias de la Salud. Universidad San Jorge.

4. Facultad de Ciencias de la Salud y del Deporte. Universidad de Zaragoza.

\section{Resumen}

Numerosos estudios evidencian que los niveles de actividad física disminuyen en la adolescencia, siendo más pronunciado este descenso entre el género femenino. Por ello, y siguiendo la teoría de la autodeterminación, el objetivo del presente estudio fue analizar la influencia del género en variables motivacionales y consecuencias afectivas y cognitivas a lo largo de diferentes unidades didácticas de Educación Física. En el estudio participaron 66 alumnos (30 varones y 36 mujeres) entre los 15 y 17 años ( $M$ edad = 15.29; DT = 0.71). Al finalizar cada unidad didáctica se midieron distintas variables motivacionales situacionales: las necesidades psicológicas básicas (BPNES), la motivación autodeterminada (EMSI), las consecuencias afectivas de diversión y aburrimiento (SSI-EF) y la predisposición hacia la práctica del contenido (PEPS). Los resultados del análisis intergrupo señalan que las chicas presentan una menor percepción de competencia que los chicos en fútbol sala $(p<.001)$. Sin embargo, las chicas perciben una mayor motivación autodeterminada, diversión y actitud cognitiva en acrosport $(p<.001)$. El análisis intra-grupo muestra en el género masculino valores superiores en las variables motivacionales en el contenido de futbol en comparación con el contenido de acrosport $(p<.05)$. Por el contrario, en el género femenino puntúan más alto en las variables motivacionales en acrosport en comparación con los otros dos contenidos de deportes colectivos de cooperación-oposición $(p<.05)$. En base a ello, se proponen directrices para reorientar el proceso de enseñanza-aprendizaje en estas unidades didácticas, mediante el desarrollo y la implementación de estrategias específicas que permitan incidir en los procesos motivacionales en función del género.

Palabras clave: motivación autodeterminada; necesidades psicológicas básicas; diversión; educación física; estrategias docentes.

\section{Abstract}

Many recent studies have reported that physical activity levels decrease during adolescence, being more pronounced among females. Therefore, based on the self-determination theory (Ryan \& Deci, 2007), the aim of the current study was to analyze the influence of gender on motivational variables and cognitive and affective consequences along different Physical Education didactic units. In this study, 66 students (30 males and 36 females), aged from 15 to 17 years, (M age $=15.29, S D=0.71$ ), were involved. At the end of the three didactic units different situational motivational variables were measured: basic psychological needs by BPNES, self-determined motivation through the EMSI, affective consequences like enjoyment and boredom using the SSI-EF and predisposition toward the practice of content by PEPS. The results show that girls have a lower perceived competence than boys in soccer $(p<.001)$. However, girls perceive a higher self-determined motivation, enjoyment and a greater cognitive attitude towards acrosport $(p<.001)$. The intra-group analysis shows higher values in soccer content compared to the contents of acrosport in boys $(p<.05)$. On the contrary, girls perceived motivational variables related to acrosport in a positive way compared to the other two contents related to cooperation-opposition sports $(p<.05)$. Therefore, guidelines are proposed to reorient the teaching-learning process in these didactic units, by developing and implementing specific strategies to influence motivational processes based on gender.

Key words: self-determined motivation; basic psychological needs; enjoyment; physical education; teaching strategies.

Correspondence/correspondencia: Luis García González

Facultad de Ciencias de la Salud y del Deporte. Universidad de Zaragoza. España

Email: Igarciag@unizar.es 
Sevil, J.; Abós, A.; Julian, J. A.; Murillo, B.; García-González, L. (2015). Género y motivación situacional en Educación Física: claves para el desarrollo de estrategias de intervención. RICYDE. Revista internacional de ciencias del deporte, 41(11), 281-296. http://dx.doi.org/10.5232/ricyde2015.04106

\section{Introducción}

$\mathrm{E}_{\mathrm{r}}^{\mathrm{n}}$ el ámbito científico existen numerosos estudios empíricos que evidencian que la práctica regular de actividad física (AF) moderada-vigorosa conlleva un gran número de beneficios saludables tanto a nivel físico, psicológico como social en todos los grupos de edad (O’Donovan y col., 2010). Todo ello es especialmente importante en la infancia y adolescencia ya que se consideran períodos críticos para la adopción de una serie de hábitos y un estilo de vida saludable y activo que puedan persistir en la transición a la etapa adulta (Telama y col., 2014). En este sentido, el entorno escolar y principalmente la asignatura de Educación Física (EF), entre otros ámbitos, juegan un papel destacado en el cumplimiento de los niveles de AF moderada-vigorosa recomendados por la Organización Mundial de la Salud (OMS) (Sallis y col., 2012). Por tanto, el docente de EF se erige como una figura prioritaria como educador, facilitador y promotor de actitudes y experiencias positivas relacionadas con la práctica y adherencia a la AF (Murillo y col., 2013).

Sin embargo, a pesar de las evidencias científicas encontradas en la literatura y las directrices que manifiesta la OMS y el curriculum vigente en favor de la práctica de AF (e.g., Dobbins, Husson, DeCorby, y LaRocca, 2013), todavía existe un alto número de personas que mantiene conductas sedentarias, originando una preocupante inactividad física entre la población. Esta tendencia es especialmente preocupante dentro del género femenino debido a que el descenso de los niveles de práctica de AF es más acusado en este colectivo (Aibar, Bois, Generelo, Zaragoza, y Paillard, 2013), mostrando niveles de AF de intensidad vigorosa a moderada inferiores al género masculino durante las clases de EF y en su vida diaria (Murillo, Julián, García-González, Albarca-Sos, y Zaragoza, 2014). Por tanto, parece necesario el diseño efectivo de estrategias de intervención para proporcionar experiencias positivas en la $\mathrm{EF}$, así como la promoción de la práctica de AF entre los adolescentes (Murillo, García, Julián, y Generelo, 2014) particularmente dentro del género femenino (Biddle, Braithwaite, y Pearson, 2014; Mitchell, Gray, y Inchley, 2013; Zook, Saksvig, Wu, y Rohm, 2014).

Una de las cuestiones más importantes a resolver entre la comunidad científica, es la comprensión de las variables que inciden en los patrones de práctica de AF entre ambos géneros. En esta línea, la perspectiva del modelo social-ecológico permite el estudio de los numerosos y diversos factores que influyen en el comportamiento y actitudes saludables y más activas relacionadas con la promoción de la AF (Eime y col., 2014), conjugando así los factores individuales (e.g., psicológicos, sociales, barreras de práctica) y los contextuales (e.g., políticas públicas saludables; Sallis, Owen, y Fisher, 2008). En este sentido, existen un gran número de trabajos que han examinado, desde perspectivas metodológicas diferentes, los factores que inciden en los bajos niveles de práctica de AF (e.g., Craggs, Corder, Van Sluijs, y Griffin, 2011). Estos autores señalan que las variables expuestas en la Teoría de la Autodeterminación (TAD; Ryan y Deci, 2007) y el modelo trans-contextual (Hagger y Chatzisarantis, 2012) permiten complementar y justificar en mayor medida la práctica de AF desde los modelos social-ecológicos.

En este sentido, y siguiendo los postulados de la TAD (Ryan y Deci, 2007) el presente estudio se centra en algunas de las variables motivacionales que pueden determinar unos mayores niveles de práctica de AF en la adolescencia. Así, la Teoría de las Necesidades Básicas (Ryan y Deci, 2002) señala que el comportamiento humano está impulsado por tres necesidades psicológicas básicas (NPB), innatas, universales y esenciales para desarrollar globalmente a la persona, y facilitar su crecimiento, así como su bienestar personal (i.e., autonomía, competencia y relaciones sociales). Atendiendo al contexto de EF, la necesidad de autonomía hace referencia al deseo de los alumnos de ser el origen de sus propias acciones 
Sevil, J.; Abós, A.; Julian, J. A.; Murillo, B.; García-González, L. (2015). Género y motivación situacional en Educación Física: claves para el desarrollo de estrategias de intervención. RICYDE. Revista internacional de ciencias del deporte, 41(11), 281-296. http://dx.doi.org/10.5232/ricyde2015.04106

(Vansteenkiste, Niemic, y Soenens, 2010). Por otro lado, la necesidad de competencia indica el deseo de los discentes de sentirse eficaces o habilidosos en las diferencias actividades planteadas (Ryan y Deci, 2007). Finalmente, la necesidad de relaciones sociales envuelve el deseo de tener intercambios comunicativos positivos, y mostrarse integrado en un grupo de iguales (Deci y Ryan, 2008). Dentro de estos mediadores psicológicos, la competencia es una de las variables más influyentes en la práctica de AF, especialmente entre las chicas (Smart y col., 2012) debido a su relación con patrones de conducta más adaptativos en la etapa adulta (Jose, Blizzard, Dwyer, McKercher, y Venn, 2011) y a la influencia de esta variable en la elección de las actividades dentro y fuera de las clases de EF (Ennis, 2011).

La satisfacción o frustración de estos tres nutrientes psicológicos, a su vez, determinan el locus de causalidad o tipo de motivación que tiene un alumno en los diferentes contextos de acción en los que participa (e.g., una actividad, una clase, un contenido curricular, las clases de EF, su estilo de vida), donde pueden aparecer formas internas, externas e impersonales. Dentro de estos niveles, un discente se encuentra motivado intrínsecamente cuando participa voluntariamente en una actividad por el mero interés, placer y satisfacción personal que obtiene practicándola (Bryan y Solmon, 2007). Sin embargo, el discente puede poseer una motivación extrínseca siempre que al participar en una actividad esté determinado por recompensas o agentes externos. En este sentido, un alumno puede realizar una actividad porque forma parte de su estilo de vida (regulación integrada), conoce los beneficios y la importancia que tiene en el desarrollo integral de la persona (regulación identificada), quiere sentirse bien y evitar sentimientos de culpabilidad o ansiedad (regulación introyectada) o quiere conseguir una recompensa externa o evitar un castigo por parte de la institución escolar (regulación externa) (Deci y Ryan, 2007). Por último, un alumno se encuentra desmotivado cuando no encuentra ningún pretexto intrínseco ni extrínseco para realizar una actividad de forma voluntaria ya que no entiende qué le aporta o qué beneficios puede obtener con ella (Ryan y Deci, 2008). En la literatura científica, existen estudios que han examinado la trayectoria de la motivación en la adolescencia, concluyendo que las formas de motivación más autodeterminadas (i.e., motivación intrínseca y regulación identificada) sufren un continuo descenso, en favor de un aumento de la motivación más controlada (e.g., Ullrich-French y Cox, 2014).

En el ámbito educativo, la satisfacción de los mediadores psicológicos y el consecuente aumento de las formas de motivación más autodeterminadas se relaciona con una serie de consecuencias afectivas (e.g., diversión) cognitivas (e.g., rendimiento académico) y comportamentales (e.g., predisposición hacia la EF) más positivas que siguen normalmente un patrón de conducta más adaptativo dentro de las clases de EF y fuera de ella (Braithwaite, Spray, y Warburton, 2011). En relación a la diversión, existen estudios longitudinales que señalan un disminución de los niveles de disfrute en EF a lo largo de la adolescencia (e.g., Yli-Piipari, Wang, Jaakkola, y Liukkonen, 2012), especialmente entre las chicas (Cairney y col., 2012). Este hecho es especialmente significativo debido a la transferencia que existe, tal y como indica el Modelo de Promoción de la AF propuesto por Welk (1999) en jóvenes, entre determinadas variables (e.g., diversión, competencia percibida, actitud cognitiva, actitud afectiva) y consecuencias como la adherencia a la práctica de AF, dentro y fuera de las clases de EF (Hilland, Ridgers, Stratton, y Fairclough, 2011; Van den Berghe y col., 2013). Algunos autores como Pieron, Castro, y González (2006) señalan la actitud del alumnado ante la EF como una variable que puede atribuirse a una mayor valoración de la asignatura. 
Sevil, J.; Abós, A.; Julian, J. A.; Murillo, B.; García-González, L. (2015). Género y motivación situacional en Educación Física: claves para el desarrollo de estrategias de intervención. RICYDE. Revista internacional de ciencias del deporte, 41(11), 281-296. http://dx.doi.org/10.5232/ricyde2015.04106

Sin embargo, la no satisfacción o frustración de estas necesidades psicológicas básicas está asociada a las formas menos autodeterminadas y a una serie de consecuencias negativas y desadaptativas en las clases de EF (e.g., aburrimiento, indisciplina, desinterés; Leptokaridou, Vlachopoulos, y Papaioannou, 2014; Sánchez-Oliva, Sánchez-Miguel, Leo, Kinnafick, y García-Calvo, 2014). En relación al aburrimiento, algunos autores como Baena-Extremera, Granero-Gallegos, Bracho-Amador, y Pérez-Quero (2012) señalan que en las clases de EF esta variable puede considerarse como un posible predictor del abandono de la práctica de $\mathrm{AF}$, siendo sus niveles más acusados en el género femenino.

De igual modo, en relación al área de EF, algunos autores han señalado que la valoración de esta asignatura disminuye conforme avanza la edad (Granero, Ruiz, y García, 2009), especialmente en el género femenino, reflejándose en una menor participación en AF de tiempo libre (Rodríguez, García-Cantó, Sánchez-López, y López-Miñarro, 2013). En relación al tipo de contenido que se imparte en el contexto de EF, chicos y chicas muestran una gran diversidad de opiniones (Gutiérrez, Pilsa, y Benet, 2007), debido, entre otros aspectos, a los estereotipos sociales imperantes en la sociedad actual (Chalabaev, Sarrazin, Fontayne, Boiché, y Clément-Guillotin, 2012). Autores como Knisel, Opitz, Wossmann, y Keteihuf (2009) señalan que los chicos están más motivados y tienen una actitud más positiva que las chicas para participar en actividades físico-deportivas, contenidos que parecen darse con mayor frecuencia en las programaciones didácticas de los docentes de EF (Robles, Abad, Castillo, Giménez y Robles, 2013). En este sentido, algunos autores demandan la importancia de dar voz activa al género femenino en la configuración de las programaciones didácticas, diversificando los diferentes contenidos curriculares (Enright y O'Sullivan, 2012; Julián, López, Aguareles, Zaragoza, y Generelo, 2008). Este se considera un aspecto trascendente para mejorar el interés y los procesos motivacionales del género femenino, adhiriendo a este colectivo a una práctica más activa en las clases de EF y fuera de ella (Kirk y Oliver, 2014).

En base al marco teórico y a los estudios previos, el objetivo del estudio fue analizar, siguiendo la TAD, la influencia del género en variables motivacionales y consecuencias afectivas y cognitivas en distintas unidades didácticas de EF. De igual modo, en base a los hallazgos encontrados, otro de los propósitos del estudio fue establecer una serie de orientaciones o estrategias didácticas específicas para cada una de estas unidades didácticas (UD), reorientando con ello el proceso de enseñanza-aprendizaje, y optimizando los procesos motivacionales en función del género. De manera paralela, teniendo en cuenta las UD sobre las que se ha llevado a cabo el estudio, se planteó como primera hipótesis que, en los deportes colectivos (fútbol, rugby), los chicos percibirían una mayor satisfacción de las NPB, una motivación más autodeterminada, y unas consecuencias afectivas y cognitivas más positivas. En sentido contrario, la segunda hipótesis sostenía que en la actividad con una finalidad artístico-expresiva (acrosport), las chicas percibirían una mayor satisfacción de las NPB, una motivación más autodeterminada, y unas consecuencias afectivas y cognitivas más positivas.

\section{Método}

\section{Normas éticas}

En lo que a las normas éticas se refiere, el estudio previamente recibió la aprobación del Comité de Ética de la Universidad de Zaragoza. Por otra parte, a nivel ético se siguieron las directrices de la Declaración de Helsinki (2008). 
Sevil, J.; Abós, A.; Julian, J. A.; Murillo, B.; García-González, L. (2015). Género y motivación situacional en Educación Física: claves para el desarrollo de estrategias de intervención. RICYDE. Revista internacional de ciencias del deporte, 41(11), 281-296. http://dx.doi.org/10.5232/ricyde2015.04106

\section{Participantes}

En el presente estudio participaron un total de 84 alumnos, distribuidos en cuatro clases de $4^{\circ}$ de Educación Secundaria Obligatoria (ESO), pertenecientes a un colegio público, con edades comprendidas entre los 15 y 17 años $(M$ edad $=15.38 ; D T=0.71)$. Tras la aplicación de los criterios de inclusión (asistencia al 85\% de las sesiones de cada UD y cumplimentación de los cuestionarios de las tres mediciones) la muestra final estuvo compuesta por 66 alumnos $(M$ $e d a d=15.29 ; D T=0.73), 30$ varones $(M$ edad $=15.28 ; D T=0.75)$ y 36 mujeres $(M$ edad $=$ $15.30 ; D T=0.78)$.

\section{Variables del estudio}

Las variables dependientes del estudio fueron las NPB (autonomía, competencia y relaciones sociales), la motivación autodeterminada (motivación intrínseca, regulación identificada, regulación externa y desmotivación), las consecuencias afectivas (satisfacción/diversión y aburrimiento) y la predisposición hacia el contenido (actitud cognitiva y actitud afectiva). Por otro lado, las variables independientes fueron el género y la UD.

\section{Instrumentos}

Escala de Medición de las Necesidades Psicológicas Básicas (BPNES). Se utilizó la versión traducida al castellano y adaptada a la EF (Moreno, González-Cutre, Chillón, y Parra, 2008) de la Escala de Medición de las Necesidades Psicológicas Básicas en el Ejercicio (BPNES; Vlachopoulos y Michailidou, 2006). Este instrumento contenía el siguiente encabezado "En las clases de la UD de ***" seguido de 12 ítems agrupados en tres factores (cuatro ítems por factor) que medían: la percepción de autonomía (e.g., "Los ejercicios que realizamos en las clases de $* * *$ se ajustan a mis intereses"), la percepción de competencia (e.g., "Realizo los ejercicios eficazmente en las clases de ***") y la percepción de relación con los demás (e.g., "Siento que me puedo comunicar abiertamente con mis compañeros en ***"). El análisis de fiabilidad obtuvo valores alpha de Cronbach en las tres mediciones realizadas de .67, .72 y .73 para la percepción de autonomía, de $.61, .67$ y .77 para la percepción de competencia y de $.73, .81$ y .86 para la percepción de relaciones sociales.

Escala de Motivación Situacional (EMSI). Se utilizó una adaptación a la EF de la versión validada al español en contexto educativo (Martín-Albo, Nuñez, y Navarro, 2009) de la Escala de Motivación Situacional (SIMS; Guay, Vallerand, y Blanchard, 2000). Las respuestas de esta escala estaban encabezadas por la pregunta "¿Por qué has participado este año en la unidad de ***?" seguidas por un total de 14 ítems agrupados en cuatro factores: cuatro ítems para la motivación intrínseca (e.g., "Porque creo que esta actividad era agradable"), tres ítems para la regulación identificada (e.g., "Porque creo que esta actividad es importante para mí"), tres ítems para la regulación externa (e.g., "Porque es algo que tengo que hacer") y cuatro ítems para la desmotivación (e.g., "No lo sé, no veo qué me aporta esta actividad"). El análisis de fiabilidad obtuvo valores alpha de Cronbach en las tres mediciones realizadas de $.84, .87$ y .82 para la motivación intrínseca, de $.83, .80$ y .71 para la regulación identificada, de $.50, .65$ y .68 para la regulación externa y de $.87, .78$ y .79 para la desmotivación. El factor de regulación identificada fue descartado del estudio debido a su baja fiabilidad en las tres mediciones, por lo que no se contempló en el posterior análisis de datos.

Escala de diversión/aburrimiento en la Educación Física (SSI-EF). Se utilizó la SSI (Duda y Nicholls, 1992), validada en el contexto español y adaptado a la EF por Baena-Extremera y col. (2012). Esta escala estaba compuesta por un total de ocho ítems agrupados en dos factores: cinco ítems medían la satisfacción/diversión (e.g., "Solía encontrar interesante las clases de ***") y tres ítems el aburrimiento (e.g., "Deseaba que las clases de *** acabasen 
Sevil, J.; Abós, A.; Julian, J. A.; Murillo, B.; García-González, L. (2015). Género y motivación situacional en Educación Física: claves para el desarrollo de estrategias de intervención. RICYDE. Revista internacional de ciencias del deporte, 41(11), 281-296. http://dx.doi.org/10.5232/ricyde2015.04106

pronto"). Los participantes respondieron a la pregunta “¿Cómo te lo has pasado en las clases de la unidad de ***?". El análisis de fiabilidad obtuvo valores alpha de Cronbach en las tres mediciones realizadas de $.86, .88$ y .89 para la satisfacción/diversión y de $.68, .70$ y .62 para el aburrimiento.

Escala de predisposición hacia la Educación Física (PEPS). Se utilizó una adaptación de la PEPS (Hilland, Stratton, Vinson, y Fairclough, 2009), traducido al castellano por un grupo de expertos, siguiendo los procedimientos habituales (Hambleton, 2005). Este instrumento fue adaptado modificando la frase inicial al contenido de la UD "En la unidad de ***". Dentro de todos los factores que componen este cuestionario se midió la actitud cognitiva y afectiva. La actitud afectiva estaba formada por tres ítems (e.g., "Las cosas que aprendo en el *** hacen que me guste más la actividad física") y la actitud cognitiva por cuatro ítems (e.g., "Las cosas que aprendo en *** me resultan útiles"). El análisis de fiabilidad obtuvo valores alpha de Cronbach en las tres mediciones realizadas de $.39, .72$ y .74 para la actitud cognitiva y de .88 , .86 y .82 para la actitud afectiva. El factor de actitud cognitiva en la primera toma fue descartado del estudio debido a su baja fiabilidad por lo que no se contempló en el posterior análisis de datos.

En el caso de los factores que han presentado una fiabilidad inferior a .70 la consistencia interna puede ser aceptada debido al pequeño número de ítems que componen el factor (Hair, Anderson, Tatham, y Black, 1998) a excepción de la regulación identificada y la actitud cognitiva en la primera toma que fueron eliminados del estudio. El formato de respuesta empleado en cada uno de los instrumentos de medida estaba indicado en una escala Likert de 1 a 5, donde el (1) correspondía a totalmente en desacuerdo y el (5) a totalmente de acuerdo con la formulación de la pregunta.

\section{Procedimiento}

Para la recogida de los datos de las diferentes variables de estudio, el investigador principal se puso en contacto con el centro de enseñanza con la finalidad de informarle de los objetivos del estudio y pedirle su consentimiento en la administración de diferentes cuestionarios. Tras la aprobación del equipo directivo, de los padres o tutores de los alumnos y del departamento de EF se realizó un estudio a lo largo de una secuencia de tres unidades didácticas (UD): fútbol sala, acrosport, y rugby, integradas en la programación didáctica anual del profesor de EF del centro.

El desarrollo del estudio se llevó a cabo a lo largo de cuatro meses, desde principios de febrero hasta finales de mayo de 2014. Las clases tuvieron una duración de 60 minutos, y una frecuencia de 2 sesiones semanales, haciendo un total de 10 sesiones de fútbol sala, 12 de acrosport y 8 de rugby. A nivel curricular las UD respetaron los objetivos, bloques de contenidos, competencias básicas y criterios de evaluación pertenecientes a $4^{\mathrm{o}}$ de ESO y vigentes en la legislación (Orden de 9 de mayo de 2007, BOA de 1 de junio de 2007). El docente de EF utilizó en las tres UD su metodología habitual, utilizando una metodología enmarcada dentro de un modelo de enseñanza comprensiva ( $O^{\prime}$ Leary, 2014).

La toma de datos se realizó al final de cada UD, destinando un tiempo de alrededor de 15 a 20 minutos para cumplimentar los diferentes cuestionarios. Para ello, se utilizó el aula de clase, en un ambiente óptimo de trabajo, con presencia del investigador principal y en ausencia del profesor de EF. 
Sevil, J.; Abós, A.; Julian, J. A.; Murillo, B.; García-González, L. (2015). Género y motivación situacional en Educación Física: claves para el desarrollo de estrategias de intervención. RICYDE. Revista internacional de ciencias del deporte, 41(11), 281-296. http://dx.doi.org/10.5232/ricyde2015.04106

\section{Análisis de datos}

De forma previa al cálculo de los resultados del estudio, se realizó un análisis de normalidad, mediante la prueba de Kolmogorov-Smirnov, obteniendo en todos las variables valores adecuados $(p>.05)$, por lo que se utilizaron pruebas paramétricas. A continuación se llevó a cabo el análisis descriptivo de todas las variables del estudio (Media y Desviación Típica). Para verificar las hipótesis planteadas se empleó un análisis de varianza multivariante MANOVA 2x3 (Género x UD) con medidas repetidas (MR) en un factor (UD). Para los contrastes multivariados se utilizaron los estadísticos Lambda de Wilks y F, junto con las comparaciones post-hoc de Bonferroni. También se calculó el tamaño del efecto mediante el valor de eta cuadrado parcial $\left(\eta_{p}^{2}\right)$ para conocer la magnitud de las diferencias encontradas, ya que elimina la influencia del tamaño de la muestra. Los distintos análisis se realizaron a través del paquete estadístico SPSS 20.0.

\section{Resultados}

En primer lugar, atendiendo al análisis inter-grupo, donde se comparan entre sí las distintas UD en función del género, se aprecia en la UD de fútbol sala un efecto significativo del género (Lambda de Wilks $\left.=.65 ; \mathrm{F}(11,54)=2.59 ; \mathrm{p}=.01 ; \eta_{\mathrm{p}}^{2}=.34\right)$, observándose en los contrastes univariados diferencias significativas en la percepción de competencia, con valores significativamente superiores para el género masculino (ver Tabla 1). En el contenido de acrosport, se obtiene un efecto significativo del género (Lambda de Wilks $=.68 ; \mathrm{F}(11,54)=$ $2.25 ; \mathrm{p}=.02 ; \eta_{\mathrm{p}}^{2}=.31$ ), cuyos contrastes univariados muestran valores significativamente superiores en el género femenino en la motivación intrínseca, diversión y actitud cognitiva, mientras que los valores de aburrimiento son significativamente superiores en el género masculino. Finalmente, en el contenido de rugby, no se encontró un efecto significativo del género $\left(\right.$ Lambda de Wilks $\left.=.77 ; \mathrm{F}(11,54)=1.39 ; \mathrm{p}=.20 ; \eta_{\mathrm{p}}^{2}=.22\right)$.

Por otro lado, en el análisis intra-grupo, donde se comparan entre sí las distintas UD en cada uno de los géneros, se observan un mayor número de diferencias (ver Tabla 1). Atendiendo al género masculino, se observa un efecto significativo de las UD (Lambda de Wilks = .37; $\left.\mathrm{F}(22,43)=3.26 ; \mathrm{p}<.00 ; \eta_{\mathrm{p}}^{2}=.62\right)$, apreciándose diferencias significativas en la autonomía, competencia, relaciones sociales, motivación intrínseca, diversión y actitud cognitiva entre los distintos contenidos de las UD, siendo los valores significativamente superiores para el contenido de fútbol sala o significativamente inferiores para el contenido de acrosport. De igual modo, aparecen valores significativamente superiores en desmotivación en el contenido de acrosport para el género masculino. En relación al género femenino, existe un efecto significativo de las UD (Lambda de Wilks $=.48 ; \mathrm{F}(22,43)=2.04 ; \mathrm{p}=.02 ; \eta_{\mathrm{p}}^{2}=.51$ ), observándose diferencias significativas en las NPB de autonomía y competencia entre los distintos contenidos de las UD con valores superiores para el contenido de acrosport. 
Sevil, J.; Abós, A.; Julian, J. A.; Murillo, B.; García-González, L. (2015). Género y motivación situacional en Educación Física: claves para el desarrollo de estrategias de intervención. RICYDE. Revista internacional de ciencias del deporte, 41(11), 281-296. http://dx.doi.org/10.5232/ricyde2015.04106

Tabla 1. Estadísticos descriptivos y análisis inter e intra-grupo en las distintas UD.

\begin{tabular}{|c|c|c|c|c|}
\hline & & $\begin{array}{l}\text { UD1-Fútbol sala (FS) } \\
\qquad M( \pm D T)\end{array}$ & $\begin{array}{c}\text { UD2-Acrosport (AC) } \\
M( \pm D T)\end{array}$ & $\begin{array}{c}\text { UD3-Rugby (RG) } \\
M( \pm D T)\end{array}$ \\
\hline \multirow{3}{*}{ AUT. } & Total & $2.58( \pm 0.60)$ & $3.02( \pm 0.64)$ & $2.99( \pm 0.60)$ \\
\hline & Masculino & $2.51( \pm 0.75)^{\mathrm{f}, \mathrm{g}}$ & $2.87( \pm 0.61)$ & $2.96( \pm 0.72)$ \\
\hline & Femenino & $2.65( \pm 0.43)$ & $3.14( \pm 0.65)^{\mathrm{h}}$ & $3.01( \pm 0.49)^{1}$ \\
\hline \multirow{3}{*}{ PC. } & Total & $3.11( \pm 0.68)$ & $3.15( \pm 0.66)$ & $3.30( \pm 0.66)$ \\
\hline & Masculino & $3.43( \pm 0.61)^{\mathrm{a}, \mathrm{j}}$ & $3.05( \pm 0.70)$ & $3.44( \pm 0.80)^{\mathrm{k}}$ \\
\hline & Femenino & $2.85( \pm 0.62)$ & $3.23( \pm 0.63)$ & $3.17( \pm 0.49)^{\mathrm{m}}$ \\
\hline \multirow{3}{*}{ RS. } & Total & $3.97( \pm 0.57)$ & $3.61( \pm 0.73)$ & $3.64( \pm 0.63)$ \\
\hline & Masculino & $4.00( \pm 0.52)^{\mathrm{n}, \mathrm{o}}$ & $3.43( \pm 0.71)$ & $3.57( \pm 0.64)$ \\
\hline & Femenino & $3.94( \pm 0.62)$ & $3.77( \pm 0.72)$ & $3.70( \pm 0.62)$ \\
\hline \multirow{3}{*}{$\begin{array}{l}\text { MOT. } \\
\text { INT. }\end{array}$} & Total & $3.20( \pm 0.97)$ & $2.87( \pm 0.90)$ & $3.17( \pm 0.74)$ \\
\hline & Masculino & $3.27( \pm 1.21)^{\mathrm{p}}$ & $2.40( \pm 0.96)$ & $3.08( \pm 0.86)^{\mathrm{q}}$ \\
\hline & Femenino & $3.14( \pm 0.72)$ & $3.26( \pm 0.64)^{b}$ & $3.25( \pm 0.63)$ \\
\hline \multirow{3}{*}{$\begin{array}{l}\text { REG. } \\
\text { EXT. }\end{array}$} & Total & $3.91( \pm 0.71)$ & $3.71( \pm 0.74)$ & $3.72( \pm 0.70)$ \\
\hline & Masculino & $3.78( \pm 0.81)$ & $3.69( \pm 0.77)$ & $3.74( \pm 0.77)$ \\
\hline & Femenino & $4.02( \pm 0.61)$ & $3.73( \pm 0.72)$ & $3.70( \pm 0.64)$ \\
\hline \multirow{3}{*}{ DESMOT. } & Total & $2.98( \pm 1.04)$ & $3.22( \pm 0.78)$ & $3.03( \pm 0.79)$ \\
\hline & Masculino & $2.74( \pm 1.31)$ & $3.37( \pm 0.84)^{\mathrm{r}}$ & $3.06( \pm 0.88)$ \\
\hline & Femenino & $3.19( \pm 0.71)$ & $3.10( \pm 0.71)$ & $3.00( \pm 0.71)$ \\
\hline \multirow{3}{*}{ DIV. } & Total & $3.48( \pm 0.85)$ & $3.28( \pm 0.81)$ & $3.47( \pm 0.75)$ \\
\hline & Masculino & $3.49( \pm 1.08)^{\mathrm{s}}$ & $2.90( \pm 0.91)$ & $3.44( \pm 0.85)^{t}$ \\
\hline & Femenino & $3.47( \pm 0.59)$ & $3.59( \pm 0.54)^{c}$ & $3.50( \pm 0.66)$ \\
\hline \multirow{3}{*}{ ABU. } & Total & $2.57( \pm 0.88)$ & $2.63( \pm 0.80)$ & $2.61( \pm 0.71)$ \\
\hline & Masculino & $2.54( \pm 1.07)$ & $2.89( \pm 0.84)^{\mathrm{d}}$ & $2.71( \pm 0.78)$ \\
\hline & Femenino & $2.58( \pm 0.71)$ & $2.41( \pm 0.70)$ & $2.52( \pm 0.64)$ \\
\hline \multirow{3}{*}{$\begin{array}{l}\text { ACT. } \\
\text { AFECT. }\end{array}$} & Total & $2.61( \pm 1.21)$ & $2.59( \pm 0.74)$ & $2.83( \pm 0.73)$ \\
\hline & Masculino & $2.39( \pm 1.08)$ & $2.42( \pm 0.82)$ & $2.88( \pm 0.86)^{\mathrm{u}}$ \\
\hline & Femenino & $2.79( \pm 1.30)$ & $2.73( \pm 0.64)$ & $2.80( \pm 0.60)$ \\
\hline \multirow{3}{*}{$\begin{array}{l}\text { ACT. } \\
\text { COGNIT. }\end{array}$} & Total & & $3.03( \pm 0.75)$ & $3.21( \pm 0.68)$ \\
\hline & Masculino & & $2.68( \pm 0.84)$ & $3.21( \pm 0.75)^{\mathrm{V}}$ \\
\hline & Femenino & & $3.33( \pm 0.50)^{\mathrm{e}}$ & $3.22( \pm 0.62)$ \\
\hline
\end{tabular}

$\mathrm{FS}=$ Fútbol sala; $\mathrm{AC}=$ Acrosport; $\mathrm{RG}=\mathrm{Rugby} ; \mathrm{AUT} .=$ Autonomía; PC.=Percepción de competencia; RS.=Relaciones sociales; MOT. INT=Motivación intrínseca; REG. EXT.= Regulación externa; DESMOT.=Desmotivación; DIV.=Diversión; ABU.=Aburrimiento; ACT. AFECT.=Actitud afectiva; ACT. COGNIT.=Actitud cognitiva.

a)Masc $>$ Fem; $p<.001 ;$ b)Fem $>$ Masc; $p<.001 ;$ c)Fem $>$ Masc; $p<.001 ;$ d)Masc $>$ Fem; $p<.05 ;$ e)Fem $>$ Masc; $p<.001 ;$ f)FS $<$ AC; $p=.05$; g)FS $<\mathrm{RG} ; p<.05 ;$ h)AC $>\mathrm{FS} ; p=.001 ; \mathrm{i}) \mathrm{RG}>\mathrm{FS} ; p<.05 ; \mathrm{j}) \mathrm{FS}>\mathrm{AC} ; p<.05 ; \mathrm{k}) \mathrm{RG}>\mathrm{AC} ; p<.05 ; 1) \mathrm{AC}>\mathrm{FS} ; p<.05 ; \mathrm{m}) \mathrm{RG}>\mathrm{FS} ; p<.05 ; \mathrm{n}) \mathrm{FS}>\mathrm{AC}$; $p<.001 ; \mathrm{o}) \mathrm{FS}>\mathrm{RG} ; p<.01 ; p) \mathrm{FS}>\mathrm{AC} ; p=.001 ; \mathrm{q}) \mathrm{RG}>\mathrm{AC} ; p=.001 ; \mathrm{r}) \mathrm{AC}>\mathrm{FS} ; p<.05 ; \mathrm{s}) \mathrm{FS}>\mathrm{AC} ; p<.01 ; \mathrm{t}) \mathrm{RG}>\mathrm{AC} ; p<.01 ; \mathrm{u}) \mathrm{RG}>\mathrm{AC} ; p<.05$; $\mathrm{v}) \mathrm{RG}>\mathrm{AC} ; p<.01$.

\section{Discusión}

El propósito del estudio fue analizar, siguiendo la TAD, la influencia del género en variables motivacionales y consecuencias afectivas y cognitivas en distintas UD de EF. Como primera hipótesis se planteó que, en los deportes colectivos (fútbol, rugby), los chicos percibirían una mayor satisfacción de las NPB, una motivación más autodeterminada, y unas consecuencias afectivas y cognitivas más positivas. En relación al análisis inter-grupo los resultados señalan que las chicas presentan valores significativamente inferiores en la percepción de 
Sevil, J.; Abós, A.; Julian, J. A.; Murillo, B.; García-González, L. (2015). Género y motivación situacional en Educación Física: claves para el desarrollo de estrategias de intervención. RICYDE. Revista internacional de ciencias del deporte, 41(11), 281-296. http://dx.doi.org/10.5232/ricyde2015.04106

competencia que los chicos en el contenido de fútbol sala, cumpliéndose parcialmente la hipótesis formulada inicialmente. Entre las posibles explicaciones a este hecho se encuentra la gran difusión que tiene el fútbol entre los chicos en edad escolar, siendo uno de los deportes más reclamados y practicados (Isorna, Rial, Vaquero, y Sanmartín, 2012). Por otro lado, la UD de rugby no presenta diferencias significativas en ambos géneros debido a que posiblemente sea un contenido menos practicado en la adolescencia. Así, los discentes no poseen unos criterios propios y externos (i.e., comparación e influencia de las opiniones de otros significativos) para tener una percepción de competencia más consolidada. Estos hallazgos son congruentes con el estudio de Murillo y col. (2014) donde se observaron, dentro de actividades de cooperación-oposición, diferencias significativas en favor del género masculino en el contenido de baloncesto y no en el de voleibol, actividad que puede tener un menor número de practicantes. De esta forma, los resultados sugieren la posibilidad de integrar contenidos más novedosos en las clases de EF, donde la percepción de competencia no está tan asentada, existiendo más oportunidades de incidir en los procesos motivacionales.

En este sentido parece un aspecto clave el apoyo a esta necesidad psicológica básica en el alumnado, a través del desarrollo de estrategias específicas de intervención, ya que la satisfacción de este mediador psicológico puede predisponer a la práctica físico-deportiva y a la adopción de estilos más activos (Craike y col., 2014). Para ello, es importante tener en cuenta estrategias didácticas que apoyen la percepción de competencia. En este sentido, los alumnos podrán plantear el calentamiento o algunas actividades a lo largo de la UD, estableciendo su propia progresión en dificultad. Asimismo, será importante que el docente de EF adapte las actividades propuestas a las características personales del alumnado, suponiendo una progresión en dificultad y un reto personal constante. De igual modo, se tratará de reforzar el error en los discentes, utilizando un feedback individual, afectivo y positivo. Así, el alumnado podrá anotar cada día un aspecto que ha mejorado en la sesión. El docente deberá tener en cuenta el progreso de los alumnos respecto a la evaluación inicial, valorando el esfuerzo y la mejora personal autoreferenciada. En esta línea, será importante regular el ritmo de aprendizaje del alumnado, planteando actividades de refuerzo y ampliación, atendiendo a sus necesidades e intereses (Jang, Reeve, y Deci, 2010; Mouratidis, Vansteenkiste, Lens, y Sideridis, 2008; Sierens, Vansteenkiste, Goossens, Soenens, y Dochy, 2009).

Asimismo, parece importante implementar en el aula las estrategias básicas en el área de EF para una implicación óptima del alumnado, detalladas por Julián, Cervelló, Del Villar y Moreno (2014) (e.g., Se desarrollarán experiencias de "éxito y fracaso controlado"), así como alguna de las orientaciones didácticas establecidas por Murillo y col. (2014) como la importancia de no relacionar las clases de EF con la práctica exclusiva de actividades competitivas. De igual modo algunos autores señalan la importancia de consultar y favorecer la toma de decisiones dentro de las clases de EF para favorecer la percepción de autonomía, competencia y relaciones sociales, permitiendo un aumento de la participación en las clases de EF (e.g., Mitchell y col., 2013). En esta línea, algunos autores señalan que resulta necesario ofrecer actividades adecuadas al nivel de habilidad de los discentes, favoreciendo oportunidades para la diversión y la relación interpersonal, y superando de esta forma las barreras que supone la práctica competitiva de algunos deportes colectivos (Brooks y Magnusson, 2007). Todo ello puede permitir superar una de los factores de práctica más importantes como es la falta de percepción de competencia dentro de algunos contenidos curriculares en las clases de EF (Mitchell y col., 2013). 
Sevil, J.; Abós, A.; Julian, J. A.; Murillo, B.; García-González, L. (2015). Género y motivación situacional en Educación Física: claves para el desarrollo de estrategias de intervención. RICYDE. Revista internacional de ciencias del deporte, 41(11), 281-296. http://dx.doi.org/10.5232/ricyde2015.04106

En relación a la segunda hipótesis, se sostuvo que en la actividad con una finalidad artísticoexpresiva (acrosport) las chicas percibirían una mayor competencia, una motivación más autodeterminada, y unas consecuencias afectivas y cognitivas más positivas. En los resultados del análisis inter-grupo las chicas presentan, en el contenido de acrosport, valores significativamente superiores en la motivación intrínseca, en la diversión, así como en la actitud cognitiva. Sin embargo, si atendemos al análisis descriptivo puede observarse que existen valores superiores en las tres NPB en relación a las chicas en el contenido de acrosport. Por otro lado, los hallazgos del análisis intra-grupo muestran diferencias significativas en las NPB de autonomía y competencia entre los distintos contenidos de las UD con valores superiores para el contenido de acrosport, por lo que la hipótesis se confirma. Asimismo, esta evidencia empírica está apoyada en el estudio de Murillo y col. (2014), que analiza la percepción de competencia en diferentes contenidos curriculares, apreciándose una mayor competencia en relación al género femenino en actividades con una finalidad artísticoexpresiva. Este hecho puede deberse a la lógica interna de este tipo de actividades donde se conjugan aspectos técnicos y colaborativos, alejados de los deportes con un carácter más competitivo como es el fútbol (Shen, Chen, Scrabis, y Tolley, 2003). Estos resultados están en línea con otros estudios que han señalado que los bloques de contenidos artísticoexpresivos, están culturalmente más asociados al género femenino, resultando más motivantes e interesantes para las chicas (Amado, Del Villar, Sánchez-Miguel, Leo, y GarcíaCalvo, 2014). Los datos obtenidos son especialmente preocupantes en los chicos, ya que la falta de disfrute puede relacionarse con al abandono de la práctica de actividad físicodeportiva (Macarro, Romero, y Torres, 2010), de ahí la importancia de generar en las clases de EF consecuencias afectivas más positivas.

En base a los resultados obtenidos, parece conveniente el desarrollo de programas de intervención multidimensional que implementen estrategias motivacionales, apoyando los tres mediadores psicológicos, en este tipo de actividades con una finalidad artístico-expresiva (ver Sevil, García-González, Julián, Abarca-Sos, y Aibar, 2014). Esto podría mejorar las formas de motivación más autodeterminadas y la diversión, logrando una mayor predisposición hacia el contenido, especialmente dentro el género masculino. Algunas de las estrategias que se han mostrado efectivas en estos contenidos con finalidad artísticoexpresiva han sido: a) posibilitar la elección de una actividad con una finalidad artísticoexpresiva, no imponiéndola; b) posibilitar la creación de pasos y actividades, así como la toma de decisiones de algunos elementos del proceso de enseñanza-aprendizaje; c) utilizar vídeos didácticos que fomenten la participación en este bloque de contenidos; d) permitir agrupaciones heterogéneas y mixtas para favorecer el apoyo y la colaboración de los compañeros; e) utilizar un feedback positivo e individual centrado en el progreso y la superación personal, evitando la frustración de sus NPB (Sevil y col., 2014).

Finalmente, como prospectiva de estudio se abre la necesidad de analizar los diferentes contenidos curriculares que atraviesa la EF escolar con el objeto de analizar cuáles son las variables motivacionales más influyentes en su práctica. En este sentido, y tras los resultados de este estudio, parece oportuno realizar programas de intervención que den soporte a los mediadores psicológicos en función del género y el contenido, para optimizar los procesos de enseñanza-aprendizaje. El análisis de las barreras de práctica en EF, a través de metodología cualitativa, y la cuantificación de los niveles de práctica, a través de acelerometría, se abren como líneas interesantes de desarrollo para optimizar las oportunidades de práctica y conseguir experiencias más positivas. En cuanto a las limitaciones del estudio es necesario señalar que se utilizó una muestra concreta en un centro educativo. Por tanto, se necesitan más evidencias empíricas que permiten generalizar los resultados obtenidos en los diferentes contenidos curriculares en las clases de EF. 
Sevil, J.; Abós, A.; Julian, J. A.; Murillo, B.; García-González, L. (2015). Género y motivación situacional en Educación Física: claves para el desarrollo de estrategias de intervención. RICYDE. Revista internacional de ciencias del deporte, 41(11), 281-296. http://dx.doi.org/10.5232/ricyde2015.04106

\section{Conclusiones}

La principal conclusión que se puede extraer de este estudio alude a la importancia de que el docente de $\mathrm{EF}$, en su intervención, desarrolle estrategias didácticas específicas para cada uno de los contenidos curriculares que pueden impartirse dentro de la EF escolar, teniendo en cuenta variables como el género. Para ello, parece muy importante evaluar, dentro de todos los factores que afectan a la $\mathrm{AF}$, las variables motivacionales, debido a su importancia para optimizar los procesos de enseñanza-aprendizaje, logrando intervenciones más individualizadas a las características e intereses propios de los discentes. Teniendo en cuenta los hallazgos encontrados en la presente investigación, parece conveniente apoyar la competencia en fútbol sala dentro del género femenino. Asimismo, parece importante generar un entorno de aprendizaje óptimo, en relación al acrosport, dentro del género masculino, para incidir en las formas de motivación más autodeterminada. Este hecho puede generar una mayor diversión y predisposición hacia la práctica del contenido. Estas directrices pueden suponer, independientemente del arraigo cultural y los estereotipos sociales, experiencias más positivas de los discentes en cada uno de los contenidos curriculares.

\section{Referencias}

Aibar, A.; Bois, J. E.; Generelo, E.; Zaragoza, J., \& Paillard, T. (2013). A cross-cultural study of adolescents' physical activity levels in France and Spain. European Journal of Sport Science, 13(5), 551-558.

http://dx.doi.org/10.1080/17461391.2012.746733

Amado, D.; Del Villar, F.; Sánchez-Miguel, P. A.; Leo, F. M., \& García-Calvo, T. (2014). Analysis of the impact of creative technique on the motivation of Physical Education students in dance content: gender differences. The Journal of Creative Behavior, O(0), 1-17. http://dx.doi.org/10.1002/jocb.69

Ames, C. (1992). Achievement goals, motivational climate, and motivational processes. In G. C. Roberts (Ed.), Motivation in sport and exercise (pp. 161-176). Champaign, IL: Human Kinetics.

Baena-Extremera, A.; Granero-Gallegos, A.; Bracho-Amador, C., \& Pérez-Quero, F. J. (2012). Spanish version of the sport satisfaction instrument (SSI) adapted to physical education. Journal of Psychodidactics, 17(2), 377-395. http://dx.doi.org/10.1387/ Rev.Psicodidact.4496

Biddle, S. J. H.; Braithwaite, R., \& Pearson, N. (2014). The effectiveness of interventions to increase physical activity among young girls: A meta-analysis. Preventive Medicine, 62, 119-131.

http://dx.doi.org/10.1016/j.ypmed.2014.02.009

Braithwaite, R.; Spray, C. M., \& Warburton, V. E. (2011). Motivational climate interventions in physical education: A meta-analysis. Psychology of Sport and Exercise, 12(6), 628-638. http://dx.doi.org/10.1016/j.psychsport.2011.06.005

Bryan, C. L., \& Solmon, M. A. (2007). Self-Determination in physical education: Designing class environments to promote active lifestyles current status of children's physical activity. Journal of Teaching in Physical Education, 26(3), 260-278.

Cairney, J.; Kwan, M. Y.; Velduizen, S.; Hay, J.; Bray, S. R., \& Faught, B. E. (2012). Gender, perceived competence and the enjoyment of physical education in children: A longitudinal examination. International Journal of Behavioral Nutrition and Physical Activity, 9(1), 26. 
Sevil, J.; Abós, A.; Julian, J. A.; Murillo, B.; García-González, L. (2015). Género y motivación situacional en Educación Física: claves para el desarrollo de estrategias de intervención. RICYDE. Revista internacional de ciencias del deporte, 41(11), 281-296. http://dx.doi.org/10.5232/ricyde2015.04106

Chalabaev, A.; Sarrazin, P.; Fontayne, P.; Boiche, J., \& Clement-Guillotin, C. (2013). The influence of sex stereotypes and gender roles on participation and performance in sport and exercise: Review and future directions. Psychology of Sport and Exercise, 14(2), 136-144. http://dx.doi.org/10.1016/j.psychsport.2012.10.005

Craggs, C.; Corder, K.; Van Sluijs, E. M. F., \& Griffin, S. J. (2011). Determinants of change in Physical Activity in children and adolescents: A systematic review. American Journal of Preventive Medicine, 40(6), 645-658. http://dx.doi.org/10.1016/j.amepre.2011.02.025

Craike, M. J.; Polman, R.; Eime, R.; Symons, C.; Harvey, J., \& Payne, W. (2014). Associations between behavior regulation, competence, physical activity, and health for adolescent females. Journal Physical Activity Health, 11(2), 410-418. http://dx.doi.org/10.1123/jpah.2012-0070.

Deci, E. L., \& Ryan, R. M. (2008). Facilitating optimal motivation and psychological wellbeing across life's domains. Canadian Psychology, 49(1), 14-23.

http://dx.doi.org/10.1037/0708-5591.49.1.14

Dobbins, M.; Husson, H.; DeCorby, K., \& LaRocca, R. L. (2013). School-based physical activity programs for promoting physical activity and fitness in children and adolescents aged 6 to 18. Cochrane Database of Systematic Reviews, 28(2) CD007651. http://dx.doi.org/10.1002/14651858.CD007651.pub2

Duda, J. L., \& Nicholls, J. G. (1992). Dimensions of achievement motivation in scholwork and sport. Journal of Educational Psychology, 84(3), 290-299. http://dx.doi.org/10.1037/0022-0663.84.3.290

Eime, R. M.; Casey, M. M.; Harvey, J. T.; Sawyer, N. A.; Symons, C. M., \& Payne, W. R. (2014). Socioecological factors potentially associated with participation in physical activity and sport: A longitudinal study of adolescent girls. Journal of Science and Medicine in Sport.

http://dx.doi.org/10.1016/j.jsams.2014.09.012

Ennis, C. D. (2011). Physical education curriculum priorities: Evidence for education and skillfulness. Quest, 63(1), 5-18. http://dx.doi.org/10.1080/00336297.2011.10483659

Enright, E., \& O'Sullivan, M. (2012). Physical Education 'in All Sorts of Corners': Student Activists Transgressing Formal Physical Education Curricular Boundaries. Research Quarterly for Exercise and Sport, 83(2), 255-267. http://dx.doi.org/10.1080/02701367.2012.10599856

Granero, A.; Ruiz, F. J., y García, M. E. (2009). Opinión de la educación física recibida. En F.J. Ruiz, M.E. García, y M. Pierón (coord.). Actividad física y estilos de vida saludable: análisis de los determinantes de la práctica en adultos (pp.151-162). Sevilla: Wanceullen.

Guay, F.; Vallerand, R. J., \& Blanchard, C. (2000). On the assessment of state intrinsic and extrinsic motivation: The situational motivation scale (SIMS). Motivation and Emotion, 24(3), 175-213.

Gutiérrez, M.; Pilsa, C., y Torres, E. (2007). Perfil de la Educación Física y sus profesores desde el punto de vista de los alumnos. Revista Internacional de Ciencias del Deporte, 8(3), 39-52.

Hagger, M. S., \& Chatzisarantis, N. L. D. (2012). Transferring motivation from educational to extramural contexts: A review of the trans-contextual model. European Journal of Psychology of Education, 27(2), 195-212.

http://dx.doi.org/10.1007/s10212-011-0082-5 
Sevil, J.; Abós, A.; Julian, J. A.; Murillo, B.; García-González, L. (2015). Género y motivación situacional en Educación Física: claves para el desarrollo de estrategias de intervención. RICYDE. Revista internacional de ciencias del deporte, 41(11), 281-296. http://dx.doi.org/10.5232/ricyde2015.04106

Hair, J. F.; Anderson, R. E.; Tatham, R. L., \& Black, W. C. (1998). Multivariate Data Analysis. New Jersey: Prentice-Hall.

Hambleton, R. K. (2005). Issues, designs and technical guidelines for adapting tests into multiple languages and cultures. En R. K. Hambleton, P. F. Merenda \& S. D. Spielberger (Eds.): Adapting educational and psychological tests for cross-cultural assessment (pp. 3-38). New Jersey: Lawrence Erlbaum Associates.

Hilland, T. A.; Stratton, G.; Vinson, D., \& Fairclough, S. J. (2009). The physical education predisposition scale: Preliminary development and validation. Journal of Sports Sciences, 27(14), 1555-1563. http://dx.doi.org/10.1080/02640410903147513

Hilland, T. A.; Ridgers, N. D.; Stratton, G., \& Fairclough, S. J. (2011). Associations between selected demographic, biological, school environmental and physical education based correlates, and adolescent physical activity. Pediatric Exercise Science, 23(1), 61-71.

Isorna, M.; Rial, A.; Vaquero, R., y Sanmartín, F. (2012). Motivaciones para la práctica de deporte federado y del piragüismo en alumnos de primaria y secundaria. Retos. Nuevas tendencias en Educación Física. Deporte y Recreación, 21, 19-24.

Jang, H.; Reeve, J., y Deci, E. L. (2010). Engaging students in learning activities: it is not autonomy support or structure but autonomy support and structure. Journal of Educational Psychology, 102(3), 588-600. http://dx.doi.org/10.1037/a0019682

Jose, K. A.; Blizzard, L.; Dwyer, T.; McKercher, C., \& Venn, A. J. (2011). Childhood and adolescent predictors of leisure time physical activity during the transition from adolescence to adulthood: a population based cohort study. International Journal of Behavioral Nutrition and Physical Activity, 8(54). http://dx.doi.org/10.1186/1479-5868-8-54

Julián, J. A.; Cervelló, E.; Del Villar, F., y Moreno, J. A. (2014). Estrategias didácticas para la enseñanza de la Educación Física. Madrid: Síntesis.

Julián, J. A.; López, N.; Aguareles, I.; Zaragoza, J., y Generelo, E. (2008). La negociación curricular en educación física cutopía o realidad?. Revista de Aula de Innovación Educativa, 173-174, 65-68.

Kirk, D., y Oliver, K. L. (2014). The same old story: the reproduction and recycling of a dominant narrative in research on physical education for girls. Apunts. Educación Física y Deportes, 116, 7-22. http://dx.doi.org/10.5672/apunts.2014-0983.es

Knisel, E.; Opitz, S.; Wossmann, M., y Keteihuf, K. (2009). Sport motivation and physical activity of students in three European schools. International Journal of Physical Education, 46, 40-53.

Leptokaridou, E. T.; Vlachopoulos, S. P., y Papaioannou, A. G. (2014). Experimental longitudinal test of the influence of autonomy-supportive teaching on motivation for participation in elementary school physical education. Educational Psychology. http://dx.doi.org/10.1080/01443410.2014.950195

Macarro, J.; Romero, C., y Torres, J. (2010). Motivos de abandono de la práctica de actividad físico-deportiva en los estudiantes de Bachillerato de la provincia de Granada. Revista Educación, 353, 495-519.

Martín-Albo, J.; Nuñez, J. L., \& Navarro, J. G. (2009). Validation of the Spanish Version of the Situational Motivation Scale (EMSI) in the Educational Context. The Spanish Journal of Psychology, 12(2), 799-807. 
Sevil, J.; Abós, A.; Julian, J. A.; Murillo, B.; García-González, L. (2015). Género y motivación situacional en Educación Física: claves para el desarrollo de estrategias de intervención. RICYDE. Revista internacional de ciencias del deporte, 41(11), 281-296. http://dx.doi.org/10.5232/ricyde2015.04106

Mitchell, F.; Gray, S., \& Inchley, J. (2013). 'This choice thing really works ...' Changes in experiences and engagement of adolescent girls in physical education classes, during a school-based physical activity programme, Physical Education and Sport Pedagogy. http://dx.doi.org/10.1080/17408989.2013.837433

Moreno, J. A.; González-Cutre, D.; Chillón, M., y Parra, N. (2008). Adaptación a la educación física de la Escala de las Necesidades Psicológicas Básicas en el Ejercicio (BPNES). Revista Mexicana de Psicología, 25(2), 295-303.

Mouratidis, A.; Vansteenkiste, M.; Lens, W., y Sideridis, G. (2008). The motivating role of positive feedback in sport and physical education: evidence for a motivational model. Journal of Sport \& Exercise Psychology, 30(2), 240-268.

Murillo, B.; García, E.; Generelo, E.; Bush, P. L.; Zaragoza, J.; Julián, J. A., \& GarcíaGonzález, L. (2013). Promising school-based strategies and intervention guidelines to increase physical activity of adolescents. Health Education Research, 28(3), 523538. http://dx.doi.org/10.1093/her/cyt040

Murillo, B.; García, E.; Julián, J. A., \& Generelo, E. (2014). Empowering adolescents to be physically active: Three- year results of the sigue la huella intervention. Preventive Medicine, 66, 6-11. http://dx.doi.org/10.1016/j.ypmed.2014.04.023

Murillo, B.; Julián, J. A.; García-González, L.; Albarca-Sos, A., y Zaragoza, J. (2014). Influencia del género y de los contenidos sobre la actividad física y la percepción de competencia en Educación Física. RICYDE. Revista internacional de ciencias del deporte, 36(10), 131-143.

http://dx.doi.org/10.5232/ricyde2014.03604

O'Donovan, G.; Blazevich, A. J.; Boreham, C.; Cooper, A. R.; Crank, H.; Ekelund, U.; ... , \& Stamatakis E. (2010). The ABC of Physical Activity for Health: a consensus statement from the British Association of Sport and Exercise Sciences. Journal of Sports Sciences, 28(6), 573-91.

http://dx.doi.org/10.1080/02640411003671212

O'Leary, N. (2014). Learning informally to use teaching games for understanding: the experiences of a recently qualified teacher. European Physical Education Review, 20(3), 367-384.

http://dx.doi.org/10.1177/1356336X14534359

Orden de 9 de mayo de 2007, del Departamento de Educación, Cultura y Deporte, por la que se aprueba el currículo de la Educación secundaria obligatoria y se autoriza su aplicación en los centros docentes de la Comunidad autónoma de Aragón, 65, 88719024. B.O.A. 1 de junio de 2007.

Piéron, M.; Castro, M. J., y González, M. A. (2006). Actitudes y motivación en Educación Física escolar. Retos. Nuevas tendencias en Educación Física, Deporte y Recreación, $10,5-22$.

Robles, J.; Abad, M. T.; Castillo, E.; Giménez, F. J., y Robles, A. (2013). Factores que condicionan la presencia de la expresión corporal en la enseñanza secundaria según el profesorado de educación física. Retos. Nuevas tendencias en Educación Física, Deporte y Recreación, 24, 171-175.

Rodríguez, P. L.; García-Cantó, E.; Sánchez-López, C., y López-Miñarro, P. A. (2013). Percepción de la utilidad de las clases de educación física y su relación con la práctica físico-deportiva en escolares. Cultura y Educación, 25(1), 65-75.

Ryan, R. M., \& Deci, E. L. (2002). An overview of self-determination theory: An organismic-dialectical perspective. In E. L. Deci y R. M. Ryan (Eds.), Handbook of self-determination research (pp. 3-33). Rochester, NY: University of Rochester. 
Sevil, J.; Abós, A.; Julian, J. A.; Murillo, B.; García-González, L. (2015). Género y motivación situacional en Educación Física: claves para el desarrollo de estrategias de intervención. RICYDE. Revista internacional de ciencias del deporte, 41(11), 281-296. http://dx.doi.org/10.5232/ricyde2015.04106

Ryan, R. M., \& Deci, E. L. (2007). Active human nature: Self-determination theory and the promotion and maintenance of sport, exercise, and health. En M. S. Hagger, y N. L. D. Chatzisarantis (Eds.), Intrinsic motivation and self-determination in exercise and sport (pp. 1-19). Champaign, IL: Human Kinetics.

Sallis, J. F.; McKenzie, T. L.; Beets, M. W.; Beigle, A.; Erwin, H., \& Lee, S. (2012). Physical education's role in public health: steps forward and backward over 20 years and HOPE for the Future. Research Quarterly for Exercise and Sport, 83(2), 125-135. http://dx.doi.org/10.1080/02701367.2012.10599842

Sallis, J. F.; Owen, N., \& Fisher, E. B. (2008). Ecological models of health behavior, in: Glanz, K., Rimer, B. K., Viswanath, K. (Eds.), Health behavior and health education: theory, research, and practice. 4th. CA: Jossey-Bass, San Francisco: 465-486.

Sánchez-Oliva, D.; Sánchez-Miguel. P. A.; Leo, F. M.; Kinnafick, F. E., y García-Calvo (2014). Physical education lessons and physical activity intentions within spanish secondary schools: A Self-Determination perspective. Journal of Teaching in Physical Education, 33, 232-249.

http://dx.doi.org/10.1123/jtpe.2013-0043

Sevil, J.; Julián, J. A.; Abarca-Sos, A.; Aibar, A., y García-González, L. (2014). Efecto de una intervención docente para la mejora de las variables motivacionales situacionales en las clases de Educación Física. Revista Retos. Nuevas tendencias en Educación Física, Deporte y Recreación, 26, 108-113.

Sierens, E.; Vansteenkiste, M.; Goossens, L.; Soenens, B., y Dochy, F. (2009). The synergistic relationship of perceived autonomy support and structure in the prediction of self-regulated learning. British Journal of Educational Psychology, 79(1), 57-68.

http://dx.doi.org/10.1348/000709908X304398.

Smart, J. E.; Cumming, S. P.; Sherar, L. B.; Standage, M.; Neville, H., \& Malina, R. M. (2012). Maturity associated variance in physical activity and health-related quality of life in adolescent females: a mediated effects model. Journal of Physical Activity and Health, 9(1), 86-95.

Telama, R.; Yang, X.; Leskinen, E.; Kankaanpa"a", A.; Hirvensalo, M.; Tammelin, T.; Viikari, J. S. A., \& Raitakari, O. T. (2014). Tracking of physical activity from early childhood through youth into adulthood. Medicine and Science in Sports and Exercise, 46(5), 955-962.

http://dx.doi.org/10.1249/MSS.0000000000000181

Ullrich-French, S., \& Cox, A. E. (2014). Normative and intra-individual changes in physical education motivation across the transition to middle school: A multilevel growth analysis. Sport, Exercise and Performance Psychology, 3(2), 132-147. http://dx.doi.org/10.1037/spy0000005

Van den Berghe, L.; Soenens, B. M.; Vansteenkiste, M.; Aelterman, N.; Cardon, G.; Tallir, I. B., \& Haerens, L. (2013). Observed need-supportive and need-thwarting teaching behavior in physical education: Do teachers' motivational orientations matter?. Psychology of Sport and Exercise, 14(5), 650-661.

http://dx.doi.org/10.1016/j.psychsport.2013.04.006

Vansteenkiste, M.; Niemiec, C., \& Soenens, B. (2010). The development of the five minitheories of self-determination theory: an historical overview, emerging trends, and future directions. In T. C. Urdan, \& S. A. Karabenick (Eds.), Advances in motivation and achievement. The decade ahead (pp. 105-166). UK: Emerald Publishing.

Vlachopoulos, S. P., \& Michailidou, S. (2006). Development and initial validation of a measure of autonomy, competence, and relatedness in exercise: The Basic Psychological Needs in Exercise Scale. Measurement in Physical Education and Exercise Science, 10(3), 179-201. 
Sevil, J.; Abós, A.; Julian, J. A.; Murillo, B.; García-González, L. (2015). Género y motivación situacional en Educación Física: claves para el desarrollo de estrategias de intervención. RICYDE. Revista internacional de ciencias del deporte, 41(11), 281-296. http://dx.doi.org/10.5232/ricyde2015.04106

Welk, G. J. (1999). The Youth Physical Activity Promotion Model: A conceptual bridge between theory and practice. Quest, 51(1), 5-23. http://dx.doi.org/10.1080/00336297.1999.10484297

Yli-Piipari, S.; Leskinen, E.; Jaakkola, T., \& Liukkonen, J. (2012). Predictive role of physical education motivation: the developmental trajectories of physical activity during grades 7-9. Research Quarterly for Exercise and Sport, 83(4), 560-569.

Zook, K. R.; Saksvig, B. I.; Wu, T. T., \& Young, D. R. (2014). Physical activity trajectories and multilevel factors among adolescent girls. Journal of Adolescent Health, 54(1), 74-80.

http://dx.doi.org/10.1016/j.jadohealth.2013.07.015. 